\title{
Equation of state measurements of hydrogen isotopes on Nova
}

G. W. Collins, L. B. Da Silva, P. Celliers, R. Cauble, D. Gold, M. Foord,

K. S. Budil, R. Stewart, N. C. Holmes, M. Ross, B. A. Hammel, J. D. Kilkenny,

$$
\text { R. J. Wallace, A. Ng* }
$$

Lawrence Livermore National Laboratory, Livermore CA 94550 USA

* University of British Columbia, Vancouver B.C.

High intensity lasers can be used to perform measurements of materials at extremely high pressures if certain experimental issues can be overcome. We have addressed those issues and used the Nova laser to shock-compress liquid deuterium and obtain measurements of density and pressure on the principal Hugoniot at pressures from $300 \mathrm{kbar}$ to more than $2 \mathrm{Mbar}$. The data are compared with a number of equation of state models. The data indicate that the effect of molecular dissociation of the deuterium into a monatomic phase may have a significant impact on the equation of state near $1 \mathrm{Mbar}$. 


\section{INTRODUCTION}

The theory of condensed molecular hydrogen predicts that at sufficiently high pressure, both the $15-\mathrm{eV}$ bandgap and the $4.5-\mathrm{eV}$ molecular dissociation energy decrease with the element, ultimately transforming into an atomic metal at $\sim 3 \mathrm{Mbar}$, even at $0 \mathrm{~K}$. Between $\sim 0.1$ and $3 \mathrm{Mbar}$, thermal dissociation and ionization can occur at temperatures well below the zeropressure limit. How hydrogen transforms from a condensed molecular state into a partially ionized dense plasma is of fundamental interest and has a profound impact on the equation of state (EOS) at high density. This EOS is integral to a broad spectrum of disciplines, such as understanding the structure of the Jovian planets or designing ignition targets for inertial confinement fusion (ICF)[1-3]. The EOS model of Saumon and Chabrier[4], which predicts that hydrogen transforms from a molecular fluid to a partially ionized atomic gas through a first-order phase transition (a so-called plasma phase transition, PPT), provides better agreement between sophisticated structural models of Saturn and measured gravitational moments than previous EOS models. The PPT has been investigated in Monte Carlo simulations[5], but most hydrogen EOS models do not predict such a phase transition. The performance of ignition ICF targets on the National Ignition Facility (NIF)[6] will rely in part on timing the breakout of a sequence of shocks to minimize the entropy production in the frozen hydrogen (deuterium-tritium) fuel during compression[7,8]. Timing these shocks depends directly on the EOS in the Mbar regime, where the molecular fluid transforms to an partially ionized and partially dissociated state. For these reasons, a number of theoretical models of the EOS of hydrogen have been proposed[4,9,10].

Hydrogen EOS data at pressures greater than 0.1 Mbar have been obtained by dynamic shock compression[11-14] and by static compression[15]. While both methods can access equilibrium 
states of matter, the final-state densities and temperatures obtained by shock compression are directly applicable to the Jovian planets and ICF. In shock compression, a single shock drives the fluid to a point on the principal Hugoniot, which is the locus of all final states of pressure, energy, and density that are achieved behind a single shock. With the initial state specified, conservation relations require only two independent parameters be measured to obtain an absolute EOS datum. The shock speed, $U_{s}$, particle speed $U_{p}$, pressure $P$, internal energy $E$, and final density $\rho$ are related by

$$
\begin{aligned}
& P-P_{o}=\rho_{o} U_{s} U_{p} \\
& \rho / \rho_{o}=U_{s} /\left(U_{s}-U_{p}\right) \\
& E-E_{o}=\frac{1}{2}\left(P+P_{o}\right)\left(\frac{1}{\rho_{o}}-\frac{1}{\rho}\right)
\end{aligned}
$$

where $\rho_{o}$ is the initial density, $P_{o}$ is the initial pressure, $\rho / \rho_{o}$ is the compression, and $E_{o}$ is the initial internal energy[16]. Equations 1-3 are the Hugoniot relations. These relations determine internal energy, not temperature, which defines how much internal energy is partitioned into kinetic energy. Temperature must be measured separately; e.g., it is often determined by measuring the optical emission.

While early shock-wave hydrogen EOS experiments[11] are well-described by an intermolecular pair potential model known as RRY[10], recent re-shock temperature measurements of Holmes et al. were found to be significantly lower than the RRY model predict[12]. These lower temperatures were attributed to the dissociation of molecules and described by a linear-mixing model based on ideal mixing of both molecular states using a softsphere perturbation theory and monatomic states using a one-component plasma model. The linear-mixing model contains one empirical parameter, which was adjusted to agree with all the 
hydrogen shock data[11,12], and predicts a significantly higher compressibility in the $0.2-$ 5.0 Mbar regime than both RRY and the widely used hydrogen Sesame tables[17].

In this paper we describe laser-driven measurements on the principal Hugoniots of liquid $\mathrm{D}_{2}$ to pressures of 2.1. Using the Nova laser[18], we compressed liquid deuterium with a laserdriven shock wave launched from an aluminum pusher. The $A l / \mathrm{D}_{2}$ interface and the shock front in the deuterium were observed with temporally resolved radiography, to determine $U_{p}, U_{s}$, and $\rho / \rho_{o}$. The pressure was calculated using (1). These absolute EOS data reveal a compressibility comparable to the linear-mixing model suggesting kinetic energy is removed from the system, perhaps through molecular dissociation. Experiments on $\mathrm{H}_{2}$ were also performed.

\section{CRYOGENIC TARGET DESIGN}

A schematic of the cryogenic target cell is shown in Fig. 1. Liquid $\mathrm{H}_{2}$ or $\mathrm{D}_{2}$ was contained in a 1.5-mm- or 1-mm-diameter, 0.45-mm-long cylindrical cell machined into a copper block. One end of the cell was sealed with an $\mathrm{Al}$ disk that served as the shock pusher; the opposite end of the cell was sealed with a 0.5 -mm-thick sapphire window. The pusher was 100,180 , or $250 \mu \mathrm{m}$ thick, depending on the experiment, with an rms surface roughness of $30 \mathrm{~nm}$. The irradiation side of the Al pusher was coated with enough polystyrene (about $20 \mu \mathrm{m}$ ) to prevent laser ablation of the $\mathrm{Al}$, minimizing $\mathrm{x}$-ray preheat of the sample. The polystyrene was overcoated with a $100-\mathrm{nm}$ layer of Al to eliminate optical laser penetration of the plastic at onset of the laser pulse before an absorbing plasma formed. To accommodate radiography through the sides of the cell, a 0.5-mm-diameter window was drilled into each side of the cell and sealed with a 5- $\mu \mathrm{m}$-thick beryllium foil. $\mathrm{D}_{2}\left(\mathrm{H}_{2}\right)$ was loaded into the cell at $\sim 20 \mathrm{~K}(15 \mathrm{~K})$ and then pressurized to a few hundred torr. Temperatures were monitored to within $0.05 \mathrm{~K}$. Initial $\mathrm{D}_{2}\left(\mathrm{H}_{2}\right)$ densities were 
determined from the saturation curve[19] to be $0.171 \mathrm{~g} / \mathrm{cm}^{3}\left(0.076 \mathrm{~g} / \mathrm{cm}^{3}\right)$. The initial density $\rho_{\mathrm{o}}$ for each experiment was known with an uncertainty of less than $0.1 \%$.

\section{EQUATION OF STATE MEASUREMENTS WITH LASERS}

It has long been known that lasers are capable of driving very strong shocks into targets[20]. However, the production of EOS data in the Mbar regime using lasers has been plagued by a number of concerns[21]. There have been four issues typically preventing accurate laser-produced EOS data: shock planarity, preheat, shock steadiness, and measurement accuracy. The experimental layout used for our EOS measurements, which addressed each of the above concerns, is shown in Fig. 2 and described below.

First, the shock produced must be planar and spatially uniform. This puts constraints on the target planarity and roughness as well as on the drive beam uniformity. One beam of the Nova laser $(\lambda=527 \mathrm{~nm})$ was focused at normal incidence onto the target, ablating the polystyrene layer and driving a shock wave through the $\mathrm{Al}$ and into the $\mathrm{D}_{2}$. A kinoform phase plate[22] was inserted into the Nova beam to smooth and produce a flat- top laser irradiance profile. The laser footprint at the target plane was elliptical, with major and minor diameters as great as 900 and $600 \mathrm{~mm}$ respectively, depending on focusing. Lineouts taken through the footprint shows speckle-to-speckle variation $\sim 15 \%$ with overall smoothness $\sim 10 \%$.

Simulations of the experiment in both one and two dimensions were done using Lasnex[23]. Evolution of the shock was determined from the mass, momentum, and energy equations and from the equation-of-state tables for each material. Simulations showed that the shock reaches a steady pressure after a few ns in the $\mathrm{Al}$ pusher. The pressure drops by about a factor of 10 as the shock unloads into the much lower density $\mathrm{D}_{2}$, and the shock remains steady 
for several ns, after which the rarefaction wave from the surface begins to overtake the shock (see Fig. 3). Two-dimensional simulations indicated that the shock front remains planar to across a diameter of several hundred microns.

Second, preheat of the sample by penetrating $\mathrm{x}$ rays or hot electrons produced in the laser-interaction region must be low. Preheat can cause an uncertainty in the initial state of the sample, which translates directly into an uncertainty in the final state. To determine the optimum ablator/pusher combination, a series of simulations and thermal expansion measurements were performed. A Michelson interferometer, sketched in Fig. 2, measured the thermal expansion of the pusher as well as shock planarity[24]. The $\mathrm{Al}$ pusher (at the $\mathrm{Al} / \mathrm{D}_{2}$ interface) forms one arm of a Michelson interferometer. If the aluminum is heated significantly before shock arrival, the sample will begin to expand, causing a shift in the fringe image. Upon shock breakout of the $\mathrm{Al}$ into the deuterium, fringes disappear due to the rapid fringe movement from the large shock velocity. The interferometer-probe beam was a 10-ns-FWHM, 355-nm laser pulse appropriately time-delayed from the Nova drive beam. Results of simulations and measurements show that a combination of a low-Z ablator and a thick (100-250 $\mu \mathrm{m}$ depending on drive) Al pusher lowered the preheat of the $\mathrm{Al} /$ deuterium interface to below $\sim 400^{\circ} \mathrm{C}$, which is the detection limit of our instrument.

Figure 4 shows the results of two different thermal expansion measurements. Figures $4 \mathrm{a}$ and $4 \mathrm{~b}$ show the breakout of aluminum pushers (see Fig. 1) during two $\mathrm{D}_{2}$ EOS experiments. These pushers were 100- $\mu$ m-thick $\mathrm{Al}$ with a 1-mm OD and coated with a $\mathrm{CH}$ ablator. Figure $4 \mathrm{a}$ shows the interferogram generated with a laser intensity of $\sim 8.5 \times 10^{13} \mathrm{~W} / \mathrm{cm}^{2}$. Motion of the $\mathrm{D}_{2} / \mathrm{Al}$ pusher interface is clearly observed beginning approximately 4 ns prior to shock breakout. Calculated shock velocities from Lasnex with this ablator/pusher combination scale with intensity 
$I$ as $\mathrm{U}_{\mathrm{s}}(\mu \mathrm{m} / \mathrm{ns}) \approx 24\left(I / 10^{14}\right)^{0.287}$; the predicted breakout occurs at $\sim 4.4$ ns after the start of the drive beam, so preheating is occurring early in the drive pulse. The shock is planar over the central $400 \mu \mathrm{m}$ of the target, with rarefaction waves moving inward from the edges causing the observed curvature. With an intensity of $1.75 \times 10^{13} \mathrm{~W} / \mathrm{cm}^{2}$, no evidence of preheating is observed, as shown in Fig. 4b. The region of planarity of the shock is $\sim 300 \mu \mathrm{m}$. .

Interferograms of the thicker (180- and $250-\mu \mathrm{m})$ pushers exhibited no fringe shifts, indicating that there was no rear-surface motion. For a detection limit of 0.2 fringe, which corresponds to movement of $30 \mathrm{~nm}$ at the pusher surface, the maximum pusher surface temperature for these targets prior to shock breakout is estimated to be $<400 \mathrm{~K}$.

Finally, shock steadiness and accurate measurements of $U_{s}, U_{p}$, and $\rho / \rho_{\mathrm{o}}$ were made with high-resolution streaked radiography of the shocked deuterium. Radiography was performed with 800-eV photons from a plasma x-ray source produced by focusing a second beam of Nova onto an Fe disk $\left(10 \mathrm{~ns}\right.$ at $\left.6 \times 10^{13} \mathrm{~W} / \mathrm{cm}^{2}\right)$. The backlighter was placed $12 \mathrm{~cm}$ from the target cell to eliminate possible heating of the cell and to produce a near-collimated source. The effective source size in the imaging direction was $\sim 150 \mu \mathrm{m}$ and was set by the width of the laser focal spot. Interferometry shows the $\mathrm{x}$-ray backlighter had no effect on the $\mathrm{D}_{2}$ in the cell. $\mathrm{X}$ rays transmitted through the target cell were imaged by a Kirkpatrick-Baez (K-B) microscope onto a streak camera. The microscope bandpass was $750-840 \mathrm{eV}$, and the collection half-angle was $2.5 \mathrm{mrad}$. Two calibrated magnifications were used: $33 \mathrm{X}$ and $82 \mathrm{X}$. The resolution of the $\mathrm{K}-\mathrm{B}$ microscope in this geometry was found to be better than $3 \mu \mathrm{m}$ over a $300-\mu \mathrm{m}$-wide field of view. The microscope imaged a strip $300 \mu \mathrm{m}$ long by 5-30 $\mu \mathrm{m}$ wide, depending on magnification and configuration. 
A streaked radiograph of shock-compressed $\mathrm{D}_{2}$ is shown in Fig. 5. The bright area in the figure is the view through the side windows of the cell. Because the pusher is opaque and the liquid transparent, the $\mathrm{Al} / \mathrm{D}_{2}$ interface is the boundary between the light and dark regions. At $1 \mathrm{~ns}$, the laser-driven shock crosses the interface, and the pusher surface accelerates to a steady speed $\left(\mathrm{U}_{\mathrm{p}}\right)$. The shock front (the dark line) precedes the interface. It is visible because of refraction of backlighter $\mathrm{x}$ rays at the density jump across the shock front. Similar to the Schlieren technique, $\mathrm{x}$ rays grazing the shock-front interface are refracted to angular deflections out of the angular field of the $\mathrm{K}-\mathrm{B}$ microscope. The steady propagation of both the shock front and the interface is demonstrated by their linear trajectories. The shock and particle speeds can be evaluated from the slopes.

The single-shock compression can be determined by Eq. (2), using the individuallyderived shock and pusher speeds. It also can be measured directly from the film as long as $U_{s}$ and $\mathrm{U}_{\mathrm{p}}$ are constant. At any time $\mathrm{t}$, the compression is equal to the ratio of two lengths: the distance between the shock front and the initial interface position, which is the thickness of a layer of uncompressed $\mathrm{D}_{2}$, and the distance between the shock front and the interface, which is the thickness of the now-compressed layer. Because all the measurements are made on one piece of film in the streak camera, uncertainties in $\rho / \rho_{o}$ due to magnification and sweep speed are canceled in this ratio. In the experiments, we observed a steady shock for 4-8 ns with no measurable change in speed of the $\mathrm{Al} / \mathrm{D}_{2}$ interface. Comparison with Eq. (2) provides an internal consistency check on $\rho / \rho_{o}$.

The shock position that we observed in the radiograph is the leading part of the shock front that emerged from the center of the pusher. In some experiments, the apparent $A 1 / D_{2}$ interface position at $\mathrm{t}=0$ on film was not identical to the actual value because cell rotation could 
be controlled only to within $3 \mathrm{mrad}$. This resulted in the center of the pusher being shadowed by an edge of the pusher before the shock front emerged from the pusher. In these cases, the shock and interface trajectories did not converge on the film. Extrapolation of the trajectories, however, revealed the actual interface position as the intersection of the two paths. The uncertainty in $\rho / \rho_{o}$ was approximately $\pm 5 \%$ for the data.

The $\mathrm{Al} / \mathrm{D}_{2}$ interface is subject to the Richtmyer-Meshkov hydrodynamic instability ( $\mathrm{R}-$ M). However, using the measured pusher finish of $30 \mathrm{~nm}$, we calculated that the largest perturbation expected from $\mathrm{R}-\mathrm{M}$ is less than $0.5 \mathrm{~mm}$ during the times of observation, approximately $1 \%$ to $2 \%$ of the compression.

\section{RESULTS AND CONCLUSION}

Figure 6 shows the pressures and final densities determined from the known initial densities and measured compressions. The error bars are governed predominantly by accuracy in determining the slopes of the shock and interface trajectories in the radiographs. The figure plots a number of $\mathrm{D}_{2}$ Hugoniot curves: the principal Hugoniot derived from the Sesame equation of state table[17]; path integral Monte Carlo (MC) simulations[5,25]; tight binding molecular dynamics (MD) simulations[26]; the plasma ACTEX model[27]; the Thomas-Fermi model QEOS[28]; and a linear-mixing model[12] referred to as the "dissociation model" in Ref. 14.

At the lowest compression, our data are in agreement with earlier gas gun results[11]; at higher compressions, the laser data deviate from most of the predictions. The data show a significantly enhanced compressibility compared to the Sesame, MD, and Thomas-Fermi predictions but similar to that of the MC results, albeit at different pressures, and the linearmixing model. The MC results also evince the so-called plasma phase transition at a pressure of a 
few hundred kbar between $\rho=0.4$ and $1.0 \mathrm{~g} / \mathrm{cm}^{3}$. The laser data are too sparse to identify this transition.

The linear-mixing model[12] most closely matches the Nova data. The main assumption in this model is that the Helmholtz free energy of an assembly of molecules and free atoms can be approximated by mixing of the free energies of pure molecular and metallic deuterium. Linear mixing is the assumption that the energy of interaction between particles of different species can be written as an average energy of interaction among the individual species. The free energy $F$ is written simply as

$$
\mathrm{F}=(1-\mathrm{x}) \mathrm{F}_{\mathrm{mol}}+\mathrm{xF}_{\mathrm{met}}-\mathrm{TS}_{\mathrm{mix}}
$$

where $F_{m o l}\left(F_{m e t}\right)$ is the molecular (metallic) free energy, $T$ is the temperature, $S$ is the entropy, and $x$ is the fraction of dissociated molecules. Minimizing the combined free energy determines the composition and the EOS of the mixture. Such a model results in an EOS that interpolates smoothly between the purely molecular and purely metallic phases.

$F_{m o l}$ is calculated using a soft-sphere variational theory[10]. $F_{m e t}$ is written in terms of a liquid metal employing the one-component plasma model. Detailed terms can be found in Ref. 12. Minimizing $F$ in (4) leads to an effective dissociation energy $D_{e}(\rho, T)$ that appears in expressions for $x$ and thermodynamic quantities derived from $F$, including the pressure $P . D_{e}$ is the energy difference between the molecular and metallic states. $D_{e}$ reduces to zero as the density becomes large, meaning that there is a greater likelihood of a transition between the two phases. $F_{m e t}$ contains a term that is difficult to calculate and in Ref. 12 was treated as an empirical parameter that was fit to gas-gun temperature data. This parameter was not adjusted for the higher pressure 
Nova data; the resulting Hugoniot is shown Fig. 6. The Hugoniot shows that energy absorbed in molecular dissociation as predicted by the model leads to lower temperatures than Sesame or the earlier RRY model in the vicinity of one Mbar for $\mathrm{D}_{2}$. A higher density is the consequence of the lower temperature. As the pressure increases along the Hugoniot, the dissociation fraction $x$ steadily increases. At the turnaround near $\rho=1 \mathrm{~g} / \mathrm{cm}^{3}, x$ is predicted to be $\approx 0.98$.

The Nova data result from the application of a technique in an EOS regime heretofore unexplored. It is highly interesting to note that not only do most of the six EOS model Hugoniots in Fig. 6 not replicate the data, but also that the various models themselves are so disparate in their predictions. The MD result[26] is similar to the much earlier Sesame prediction[17]; both barely achieve a compression larger than the single-shock, ideal monatomic gas limit of 4 , while the ideal diatomic compression is 8. (The laser data show a maximum compression of about 6.) ACTEX, which utilizes an expansion in the activity and is a theory suitable for fully-to-partially ionized plasmas, predicts a compression of 6 at $1.7 \mathrm{Mbar}$, but the model is unstable at pressures below this value[27]. In low-temperature applications such as shock compression, ACTEX can be sensitive to changes in potentials between neutral particles, but it accurately describes high pressure shock data for a number of materials[29].

Results for $\mathrm{H}_{2}$ were similar to those for $\mathrm{D}_{2}$.

There are two important consequences of these experiments and the predicted enhanced compressibility of hydrogen isotopes. First, higher compressibility suggests that the mass distribution in the Jovian planets is different than previously thought. Second, the more compressible EOS of hydrogen leads directly to higher fuel densities in ICF targets; this offers the possibility of higher performance and improved margin for NIF ignition capsules. 
In conclusion, these experiments demonstrate that laser-driven shocks can effectively and confidently be used for EOS studies at pressures beyond those attainable by traditional techniques. It is expected that the data presented here will stimulate theoretical work on hydrogen isotopes at high energy density.

\section{ACKNOWLEDGMENTS}

The authors would like to thank W. J. Nellis, M. Ross, F. J. Rogers, and D. A. Young for highly informative discussions. This work was performed under the auspices of the U.S. Department of Energy by Lawrence Livermore National Laboratory under contract no. W-7405-Eng-48. 


\section{REFERENCES}

1. S. Ichimaru, H. Iyetomi, and S. Tanaka, Phys. Rep. 149, 91 (1987).

2. N. W. Ashcroft, Phys. World 8(7), 43 (1995).

3. R. Smoluchowski, Nature 215, 691 (1967); V. N. Zharkov and V. P. Trubitsyn, in Jupiter, T. Gehrels, Ed. (University of Arizona Press, Tucson, 1976), pp. 135-175; W. B. Hubbard, Science 214, 145 (1981); W. J. Nellis, M. Ross, and N. C. Holmes, Science 269, 1249 (1995).

4. D. Saumon, G. Chabrier, and H. M. Van Horn, Astrophys. J. Supp. 99, 713 (1995) and references therein.

5. W. R. Magro, D. M. Ceperley, C. Pierleoni, and B. Bernu, Phys. Rev. Lett. 76, 1240 (1996).

6. J. A. Paisner, E. M. Campbell, and W. J. Hogan, Fusion Technology 26, 755 (1994).

7. J. D. Lindl, Phys. Plasmas 2, 3933 (1995).

8. S. W. Haan, S. M. Pollaine, J. D. Lindl, L. J. Suter, R. L. Berger, L. V. Powers, W. E. Alley, P. A. Amendt, J. A. Futterman, W. K. Levedahl, M. D. Rosen, D. P. Rowley, R. A. Sacks, A. I. Shestakov, G. L. Strobel, M. Tabak, S. V. Weber, G. B. Zimmerman, W. J. Krauser, D. C. Wilson, S. V. Coggeshall, D. B. Harris, N. M. Hoffman, and B. H. Wilde, Phys. Plas. 2, 2480 (1995); W. J. Krauser, N. M. Hoffman, D. C. Wilson, B. H. Wilde, W. S. Varnum, D. B. Harris, F. J. Swenson, P. A. Bradley, S. W. Haan, S. M. Pollaine, A. S. Wan, J. C. Moreno, and P. A. Amendt, Phys. Plas. 3, 2084 (1996).

9. W. B. Hubbard, Astrophys. J. 152, 745 (1968).

10. M. Ross, F. H. Ree, and D. A. Young, J. Chem. Phys. 79, 1487 (1983). 
11. W. J. Nellis, A. C. Mitchell, M. van Theil, G. J. Devine, R. J. Trainor, and N. Brown, J. Chem. Phys. 79, 1480 (1983).

12. N. C. Holmes, M. Ross, and W. J. Nellis, Phys. Rev. B 52, 15835 (1995).

13. S. T. Weir, A. C. Mitchell, and W. J. Nellis, Phys. Rev. Lett. 76, 1860 (1996).

14. L. B. Da Silva, P. Celliers, G. W. Collins, K. S. Budil, N. C. Holmes, T. W. Barbee, Jr., B. A. Hammel, J. D. Kilkenny, R. J. Wallace, M. Ross, A. Ng, G. Chiu, and R. Cauble, Phys. Rev. Lett. 78, 483 (1997); R. Cauble, L. B. Da Silva, T. S. Perry, D. R. Bach, K. S. Budil, P. Celliers, G. W. Collins, A. Ng, T. W. Barbee Jr, B. A. Hammel, N. C. Holmes, J. D. Kilkenny, R. J. Wallace, G. Chiu, and N. C. Woolsey, Phys. Plasmas 4, 1857 (1997).

15. H. K. Mao and R. J. Hemley, Rev. Mod. Phys. 66, 671 (1994); P. Loubeyre, R. LeToullec, D. Haussermann, and D. Hanfland, Nature 383, 702 (1996).

16. Y. B. Zel'dovich and Y. P. Raizer, Physics of Shock Waves and High-Temperature Hydrodynamic Phenomena (Academic Press, New York, 1966).

17. See National Technical Information Service document No.LA-4776 (G. I. Kerley, “A Theoretical Equation of State for Deuterium," 1972). Copies may be ordered from the National Technical Information Service, Springfield, VA 22161.

18. E. M. Campbell, Laser Part. Beams 9, 209 (1991).

19. P. C. Souers, Hydrogen Properties for Fusion Energy (University of California Press, Berkeley, 1986).

20. R. J. Trainor, J. W. Shaner, J. M. Auerbach, and N. C. Holmes, Phys. Rev. Lett. 42, 1154 (1979).

21. See National Technical Information Service document No. UCRL-LR-105820-93 (R. Cauble, L. B. Da Silva, S. G. Glendinning, S. M. Lane, T. S. Perry, and D. W. Phillion, “Techniques 
for Mbar to Near-Gbar Equation-of-State Measurements with the Nova Laser," in Inertial Confinement Fusion, 1993 ICF Annual Report, 1993, pp 131-136). Copies may be ordered from the National Technical Information Service, Springfield, VA 22161.

22. S. N. Dixit, M. D. Feit, M. D. Perry and H. T. Powell, Opt. Lett. 21, 1715 (1996).

23. G. B. Zimmerman and W. L. Kruer, Comments Plas. Phys. 2, 51 (1975).

24. See National Technical Information Service document No. UCRL-LR-105821-97-1 (K. S. Budil, P. Celliers, G. W. Collins, L. B. Da Silva, R. Cauble, R. J. Wallace, and G. Chiu, “Characterization of Laser-Driven Shock Waves Using Interferometry," in ICF Quarterly, 1997, pp. 11-15). Copies may be ordered from the National Technical Information Service, Springfield, VA 22161.

25. B. Militzer, W. Magro, and D. Ceperley, "Fermionic Path Integral Simulation of Dense Hydrogen," Proceedings of the 1997 International Conference on Strongly Coupled Coulomb Systems (Boston), to be published in 1998.

26. T. J. Lenosky, J. D. Kress, and L. A. Collins, Phys. Rev. B 56, 5164 (1997).

27. F. J. Rogers, Astrophys. J. 310, 723 (1986); F. J. Rogers, F. J. Swenson, and C. A. Iglesias, Astrophys. J. 456, 902 (1986).

28. R. M. More, K. H. Warren, D. A. Young, and G. B. Zimmerman, Phys. Fluids 31, 3059 (1988).

29 F. J. Rogers and D. A. Young, "Validation of the Activity Expansion Method (ACTEX) with Ultrahigh Pressure Shock Equations of State," to appear in Phys. Rev. E (1997). 


\section{Figure Captions}

Fig. 1. Diagram of the cryogenic cell used in the EOS experiments. The Nova drive beam is incident on an aluminum pusher through which a shock propagates and unloads into a container of liquid $\mathrm{D}_{2}$. Backlighter $\mathrm{x}$ rays transit the package through Be-covered holes in the sides. A probe laser for interferometry views the pusher surface through a sapphire window.

Fig. 2. Schematic diagram of the experimental setup for simultaneous side-on radiography and end-on interferometry of a cryogenic EOS target.

Fig. 3. Simulated $\mathrm{D}_{2}$ pressure vs distance at 1-ns intervals for a laser intensity of 2-×-14$\mathrm{W} / \mathrm{cm}^{2}$ incident on a $180-\mu \mathrm{m}$-thick $\mathrm{Al}$ pusher. The small variations in pressure are due to zoning in the simulation.

Fig. 4. Streak interferograms of the rear surface of two shocked $100-\mu$ m-thick Al pushers. In

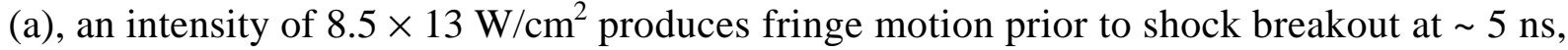
indicating expansion of the surface caused by x-ray preheat. In (b), a lower intensity, $1.75 \times 10^{13} \mathrm{~W} / \mathrm{cm}^{2}$, shows no movement.

Fig. 5. A side-on streak radiograph of shocked liquid $\mathrm{D}_{2}$. The shock moves from left to right. The $\mathrm{Al} / \mathrm{D}_{2}$ interface is the boundary between light and dark regions. The shock front is the dark line leading the interface.

Fig. 6. Nova data (open squares) and gas gun data[11] (open circles) compared with predicted $\mathrm{D}_{2}$ principal Hugoniots: Sesame[17] (solid line); path integral Monte Carlo simulations[5,25] (dash-double-dot); tight binding molecular dynamics simulations[26] (dots); ACTEX model[27] (long dashes); QEOS[28] (dash-dot); and a linear-mixing model[12] (short dashes). 


\section{Drive}

\section{Beam}

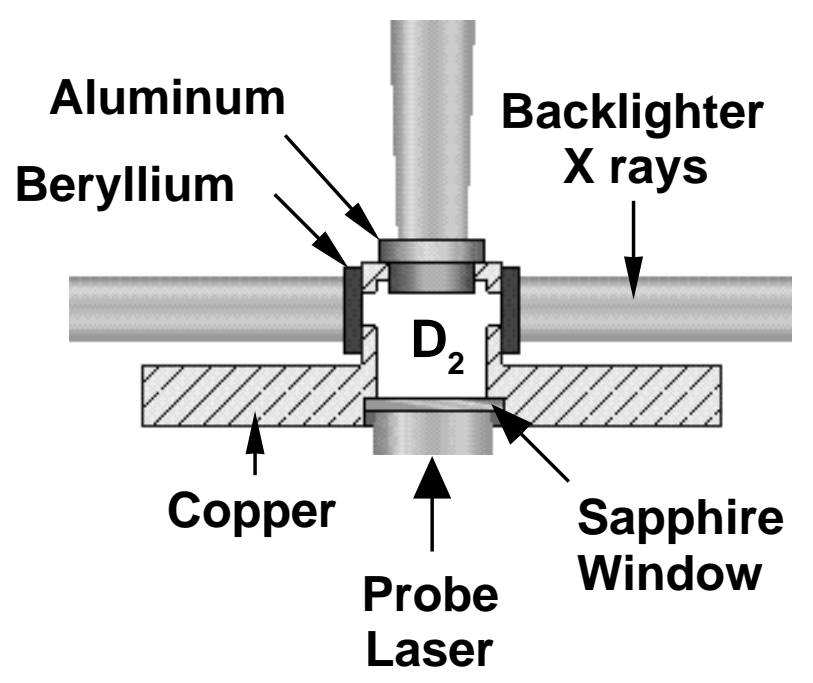

Collins Fig 1 


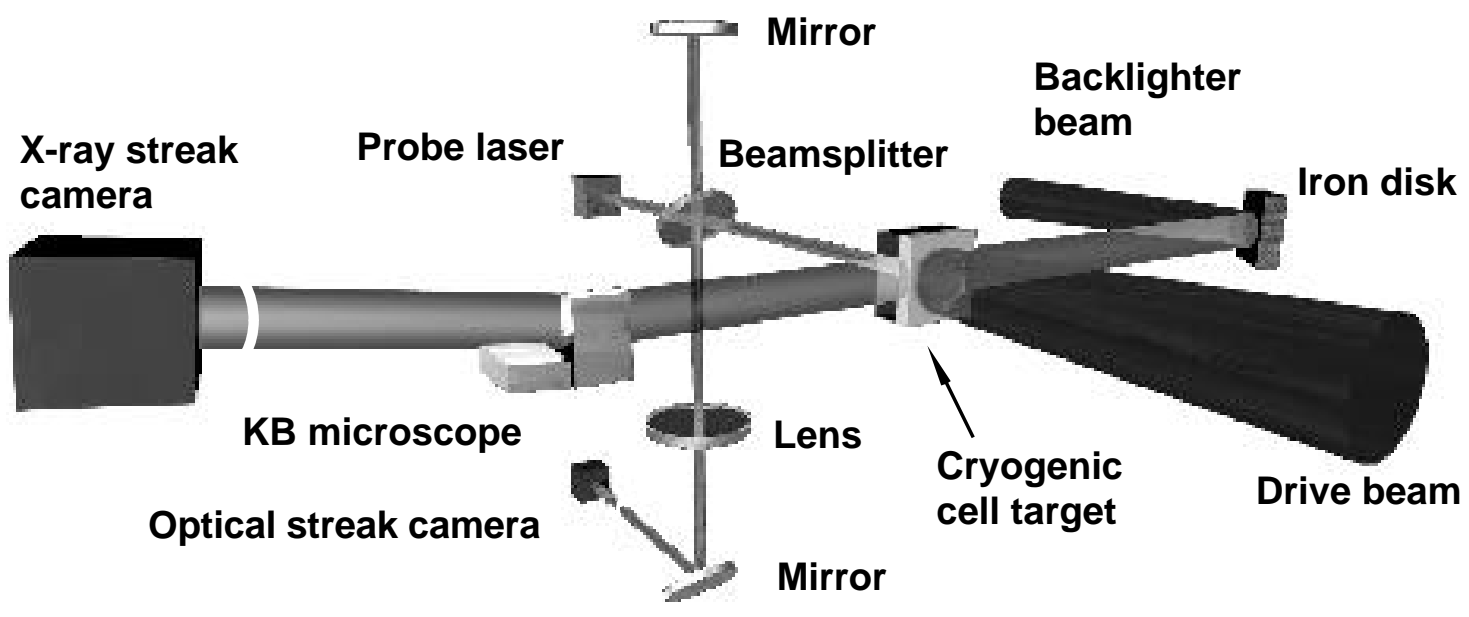

Collins Fig 2 


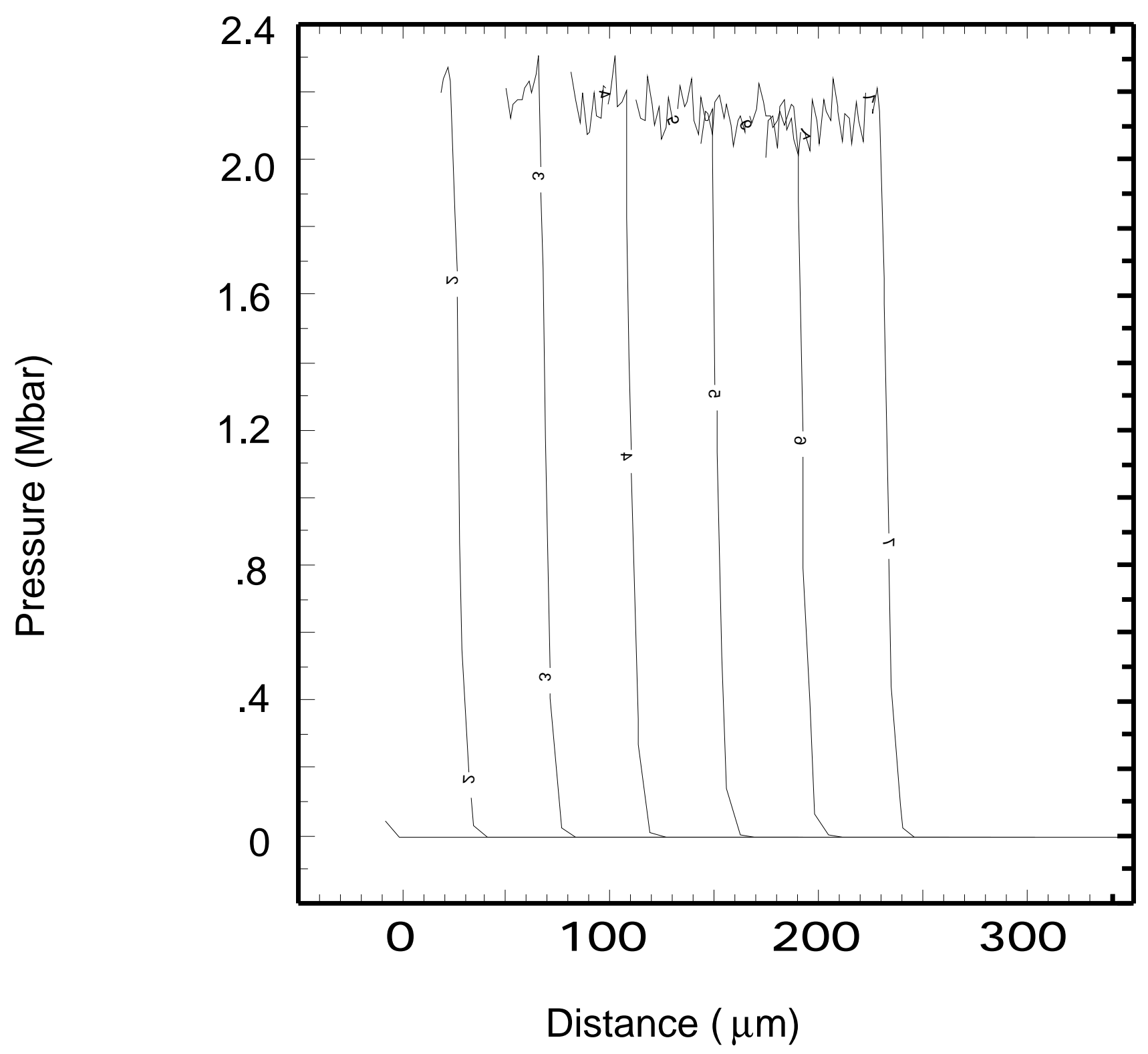

Collins Fig 3 


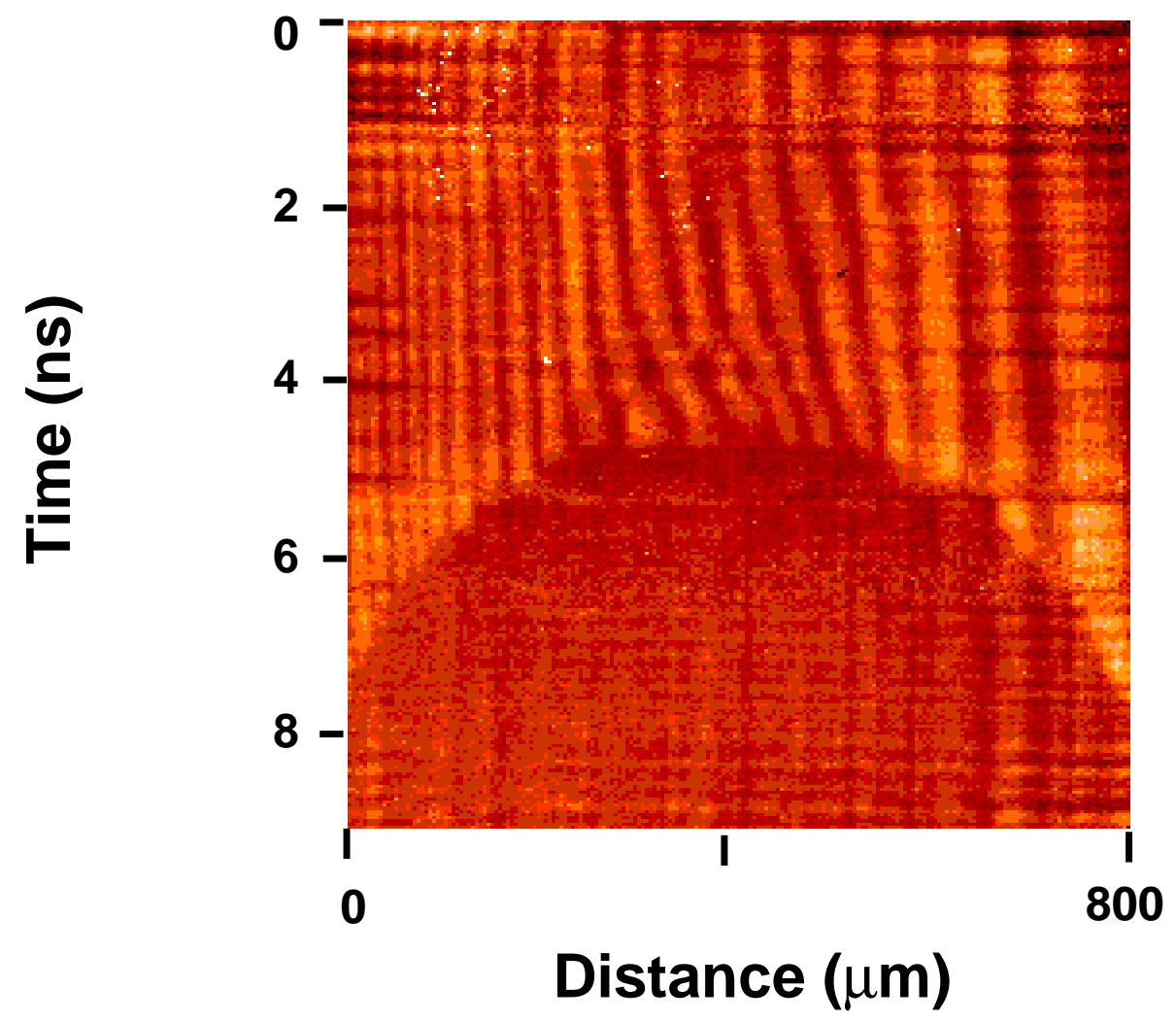

Collins Fig $4 a$

to be printed in $B \& W$ - not color 


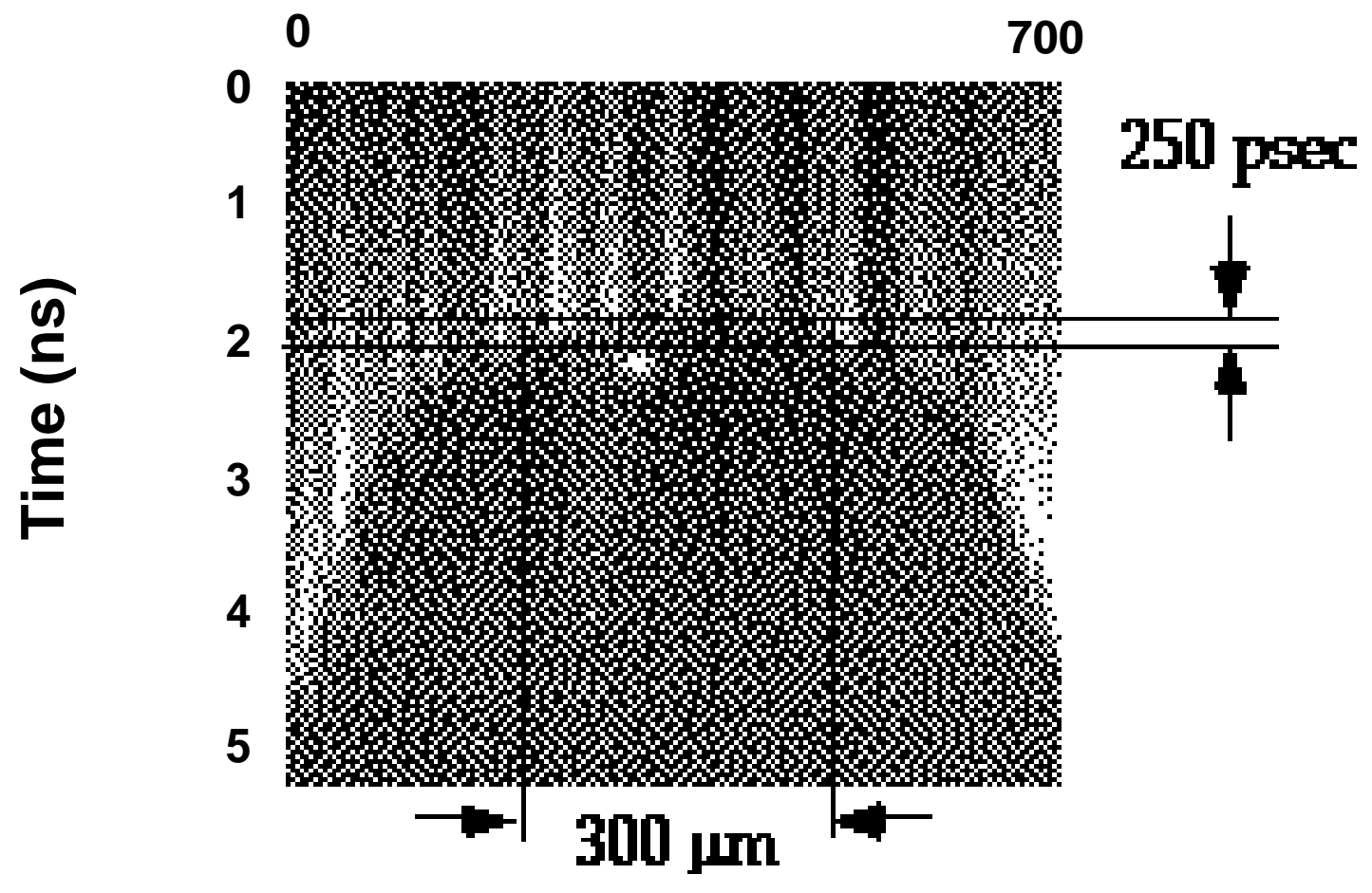

Distance $(\mu \mathrm{m})$ 


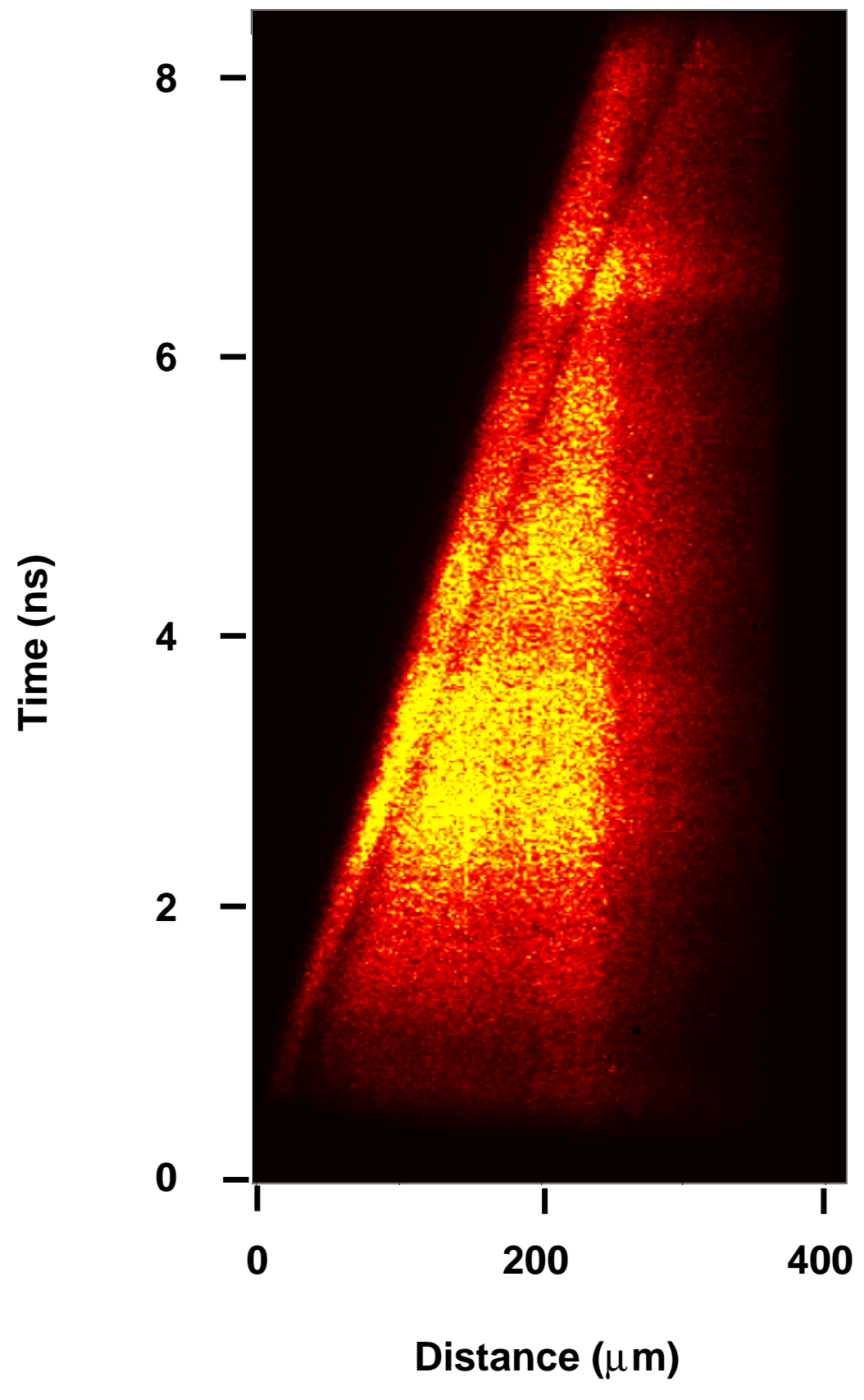

Collins Fig 5

to be printed in B\&W -- not in color 


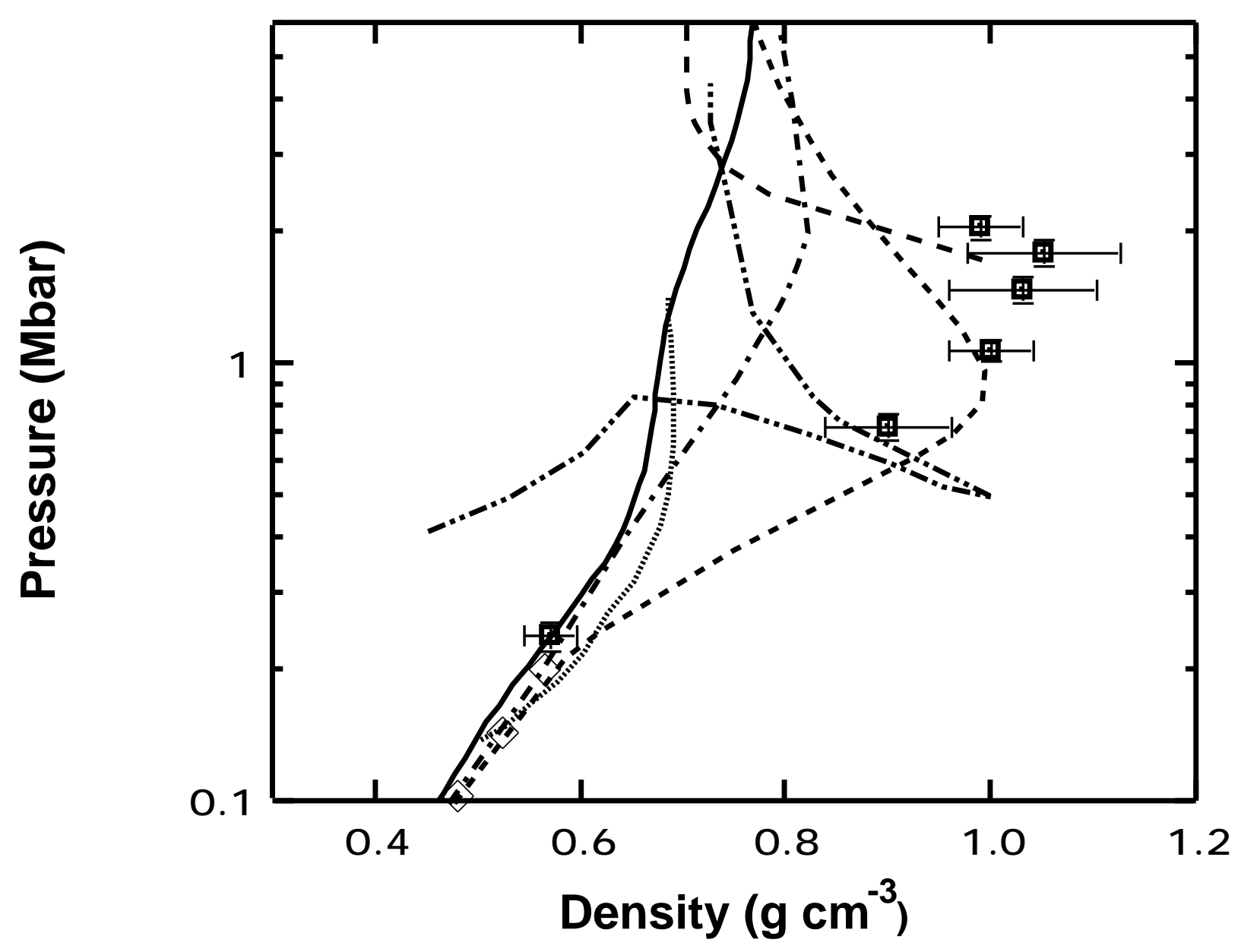

Collins Fig 6 